\title{
Infrarenal abdominal aortic aneurysm rupture and severe symptomatic coronary artery disease: Rapid combined transdiaphragmatic off-pump coronary surgery and abdominal aortic aneurysm repair
}

\author{
Georges Fayad, MD, ${ }^{\text {a }}$ Thomas Modine, MD, ${ }^{a}$ Stephan Haulon, MD, ${ }^{\mathrm{b}}$ Christophe Decoene, MD, ${ }^{\text {a }}$ Richard Azzaoui, MD, \\ Reza Salari, MD, and Mohamed Koussa, MD, ${ }^{\mathrm{b}}$ Lille, France
}

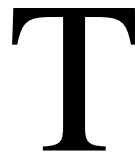
he incidence of severe coronary disease in patients with abdominal aortic aneurysms (AAAs) is about 30\%. Impaired myocardial performance in patients undergoing vascular reconstruction is the first cause of morbidity and mortality, especially in cases of ruptured AAA. Coronary revascularization is often not performed because of the necessity of a rapid aortic reconstruction. We describe a rapid combined aortic and coronary procedure to reduce postoperative cardiac events.

\section{Clinical Summary}

A 76-year-old man who had thoracic pain at rest and an 85-mm maximum diameter infrarenal AAA on helical computed tomography underwent a cardiac evaluation. Echocardiography revealed a $35 \%$ ejection fraction, together with inferior hypokinesia. A thallium myocardial scintigraphy depicted severe inferior ischemia. Coronary angiogram revealed a normal left coronary network and a very tight stenotic lesion of the midright coronary artery (Figure 1, $A$ ). An angioplasty was attempted without success (Figure 1,B).

An endovascular exclusion of the AAA was ruled out because of a short $(<15 \mathrm{~mm})$ infrarenal neck. While hospitalized and awaiting myocardial revascularization, the patient presented with a sudden onset of abdominal pain. His hemoglobin rate decreased from 14.8 to $10.7 \mathrm{~g} / \mathrm{dL}$. He was immediately brought to the operating room.

A horizontal subcostal laparotomy was performed, and the internal saphenous vein was harvested. The celiac aorta was exposed (Figure 2, A), and a 7-cm horizontal incision of the diaphragm was made to obtain a good exposure of the posterior interventricular artery. Heart exposure and stabilization were achieved by using the octopus 4-coronary stabilizer (Medtronic, Inc, Minneapolis, Minn).

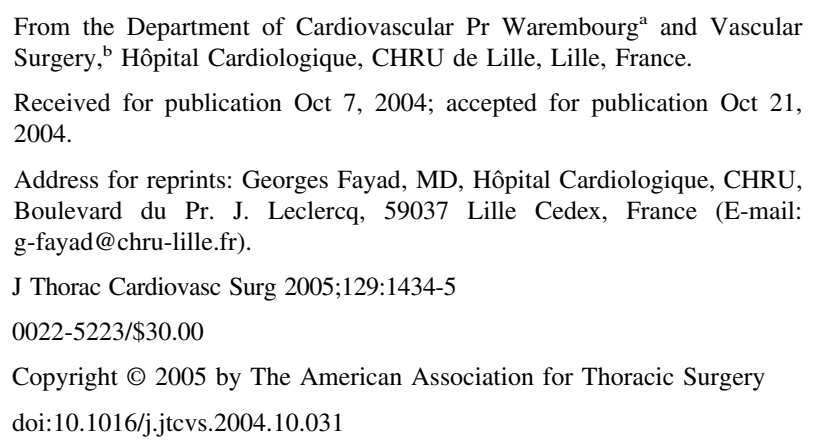

Heparin (100 IU/kg) was then administered. The coronary vessel was seized above the anastomotic site with a polytetrafluoroethylene (Gore-Tex; W. L. Gore \& Associates, Inc, Flagstaff, Ariz) 4-0 suture to prevent coronary artery injury. An end-to-side anastomosis between the vein graft and the posterior interventricular artery was achieved. The proximal side-to-end anastomosis between the celiac aorta and the vein graft was performed during partial celiac aorta clamping (Figure 2,B).

After standard closure of the diaphragm (Figure 2, B), we approached the AAA. An extensive retroperitoneal hematoma was detected. A standard AAA resection was performed with a bifurcated graft $(24 \times 12 \mathrm{~mm})$. No postoperative myocardial infarction or major cardiac complications were depicted (troponin at 0.71 $\mathrm{ng} / \mathrm{mL}$; standard troponin, $<0.6 \mathrm{ng} / \mathrm{mL}$ ).

On postoperative day 2 , the patient experienced septic shock. Extubation was finally carried out on day 40, but the patient died 10 days later in a physiologic state of depletion.

\section{Discussion}

Combination of AAA and coronary artery disease is common. Currently, there seems to be a consensus in favor of combined surgical intervention to reduce perioperative mortality. ${ }^{1,2}$

According to Ascione and associates, ${ }^{3}$ it appears that off-pump coronary surgical procedures decrease postoperative complications in high-risk patients undergoing simultaneous coronary and abdominal aortic operations compared with the conventional 1-stage procedure.

In this high-risk patient older than 75 years and despite the emergency caused by the rupture, we could not eliminate myocardial revascularization because of the severe symptomatic coronary artery disease associated with a $35 \%$ ejection fraction and the inferior ischemia observed during myocardial scintigraphy. ${ }^{4}$

Our decision was to perform a combined operation while avoiding 2 major morbidity risk factors, which are extracorporeal circulation and sternotomy in a patient having respiratory insufficiency.

The absence of extracorporeal circulation allowed us to avoid a substantial anticoagulation at $300 \mathrm{IU} / \mathrm{kg}$ on a ruptured aneurysm. Furthermore, off-pump surgical intervention prevents complications of cardiopulmonary bypass.

The absence of sternotomy is beneficial to the respiratory process and reduces postoperative pain.

Unlike the technique described by Kameda and colleagues ${ }^{5}$ concerning 4 patients with stable hemodynamics, we chose to use a saphenous vein graft instead of a gastroepiploic artery because of the necessity of performing an emergency procedure. The advantage rests in the easy harvesting achieved 


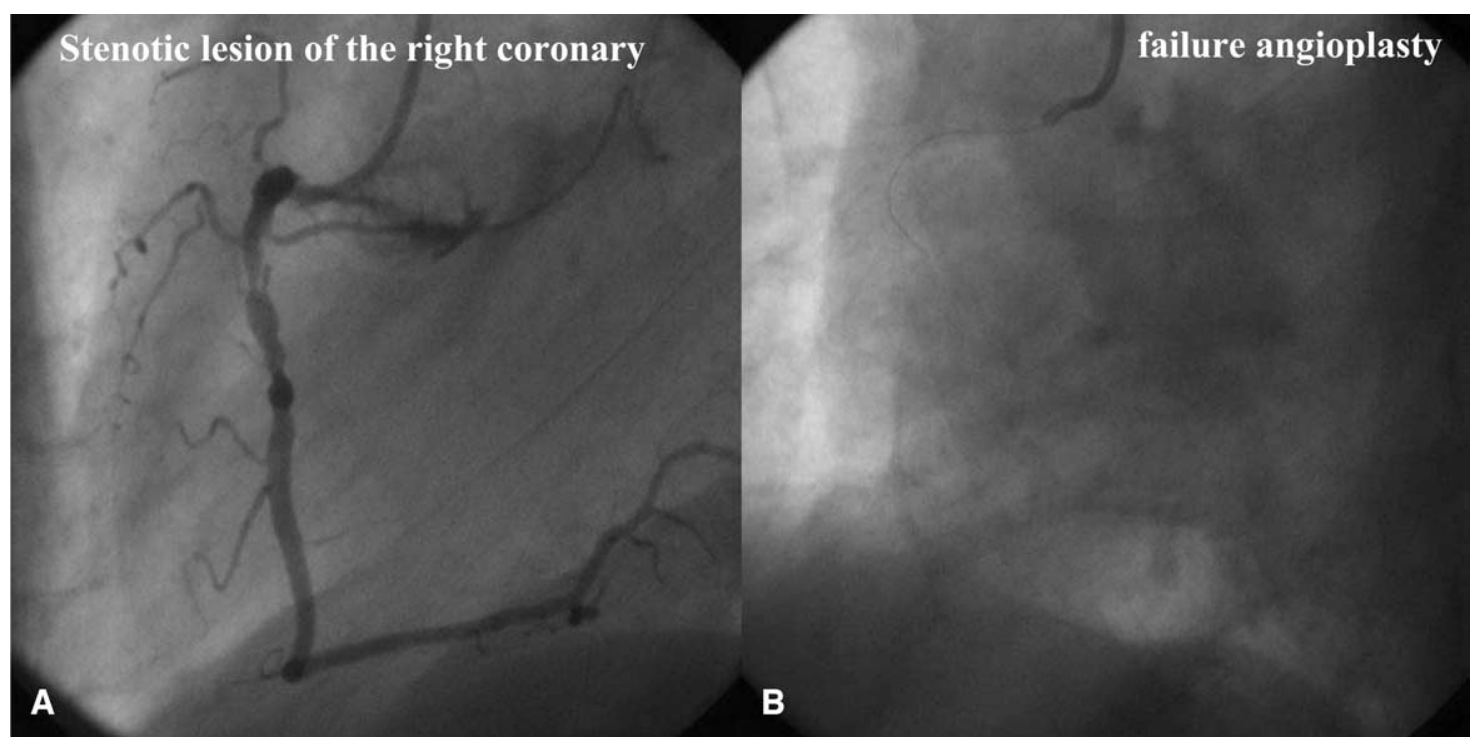

Figure 1. Coronary arteriographic view of the right coronary artery.

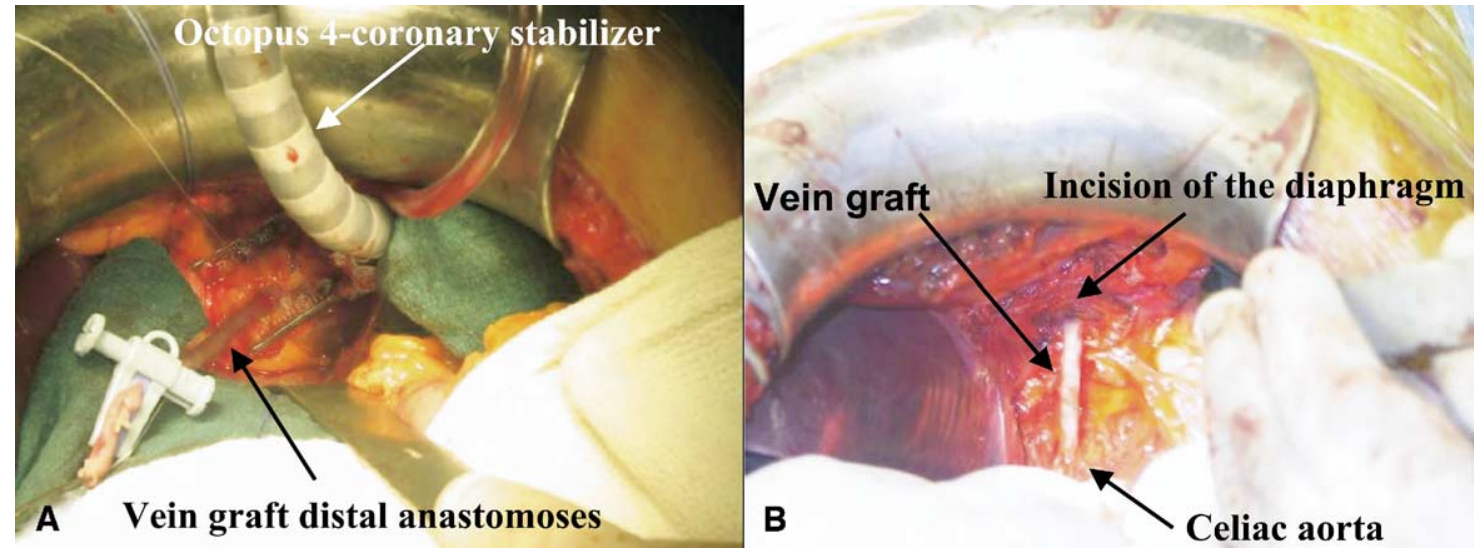

Figure 2. Intraoperative view.

simultaneously with the laparotomy. The entire cardiac procedure lasted about 30 minutes.

\section{Conclusion}

The interesting aspect of this case lies in the combined management of a ruptured aneurysm and a beating-heart coronary bypass performed through a laparotomy and on the graft choice and its implantation. It is an easy and reproducible procedure to perform in dedicated indications.

\section{References}

1. Friedman SG, Safa T, Nussbaum T, Pogo G, Levy M. Combined off-pump coronary artery bypass and abdominal aortic surgery is asso- ciated with low morbidity and mortality. Ann Vasc Surg. 2003;17: 162-4.

2. Gade PV, Ascher E, Cunningham JN, Kallakuri S, Scheinman M, $\mathrm{Scherer} \mathrm{H}$, et al. Combined coronary artery bypass grafting and abdominal aortic aneurysm repair. Am J Surg. 1998;176:144-6.

3. Ascione R, Ianelli G, Lim KHH, Imura H, Spampinato N. One-stage coronary and abdominal aortic operation with or without cardiopulmonary bypass: early and mid-term follow-up. Ann Thorac Surg. 2001;72: $768-75$.

4. Mohr FW, Falk V, Autschbach R, Diegeler A, Schorn B, Weyland A, et al. One-stage surgery of coronary arteries and abdominal aorta in patients with impaired left ventricular function. Circulation. 1995;91: 379-85.

5. Kameda Y, Shigeki T, Kawata T, Tabayashi N, Kimura M. Minimally invasive direct coronary artery bypass combined with abdominal aortic aneurysm repair. Ann Thorac Surg. 1999;68:1537-9. 